\title{
Testosterone-Induced Changes in Markers of Bone Turnover in Adolescent Boys with Testicular Dysfunction
}

\author{
Eishin Ogawa, Yuriko Katsushima, Ikuma Fujiwara and Kazuie linuma \\ Department of Pediatrics, Tohoku University School of Medicine, Sendai, Japan
}

\begin{abstract}
The effects of testosterone (T) for pubertal induction on bone mineral density (BMD) and biochemical markers of bone turnover were evaluated in three boys with testicular dysfunction. Testosterone enanthate (50 mg) was administered every 4 weeks for 1 year intramuscularly in all subjects. BMD and markers of bone turnover including serum osteocalcin, bone-specific alkaline phosphatase (bALP), urinary deoxypyridinoline (Dpyr), and N-terminal telopeptide of type I collagen, were measured before and during T treatment. BMD steadily increased during treatment. All markers of bone turnover were increased at 6 months of treatment, although increases were statistically significant only in bALP and Dpyr. An initial increase in markers of bone turnover has not been described in adults, but the observed changes resemble spontaneous puberty. The effects of exogenous $\mathrm{T}$ administration on bone turnover appear to differ depending on age.
\end{abstract}

Key words: testosterone, hypogonadism, bone mineral density, bone turnover markers

\section{Introduction}

Hypogonadism is accompanied by low bone mineral density (BMD) (1), and testosterone (T) replacement therapy resulted in a steady increase in BMD in hypogonadal adolescent boys and adult men (2-5). Several markers of bone turnover have been utilized to investigate the mechanisms for increased BMD by T treatment; these suggest that reduced bone resorption is considerable in adults $(3,4)$ and is largely due to the effects of estrogens converted from $\mathrm{T}(6)$. Nevertheless, during puberty when BMD increases with the T

Received: March 6, 2003

Accepted: September 8, 2003

Correspondence: Dr. Eishin Ogawa, Department of Pediatrics, Tohoku University School of Medicine, 1-1 Seiryo-machi, Aoba-ku, Sendai 980-8574, Japan.

E-mail: eogawa@ped.med.tohoku.ac.jp increment, markers for both bone formation and resorption are initially increased, and then decrease into the adult range $(7-11)$. Yet, the effects of exogenous $\mathrm{T}$ treatment on bone turnover markers in a pubertal age group have not been described. To clarify this issue, we measured serum levels of osteocalcin (OC) and bone-specific alkaline phosphatase (bALP) as markers of bone formation and urinary deoxypyridinoline (Dpyr) and N-terminal telopeptide of type I collagen (NTx) as markers of bone resorption before and during $\mathrm{T}$ replacement therapy in adolescent hypogonadal boys.

\section{Patients and Methods}

\section{Patients}

Three adolescent boys aged between 14.2 and $16.5 \mathrm{yr}$ with testicular dysfunction of various 
Table 1 Demographics

\begin{tabular}{lccc}
\hline & Case 1 & Case 2 & Case 3 \\
\hline Age & 16 y $6 \mathrm{~m}$ & 14 y $2 \mathrm{~m}$ & $16 \mathrm{y} 4 \mathrm{~m}$ \\
Height $(\mathrm{cm})$ & 158.5 & 148.0 & 147.6 \\
Weight $(\mathrm{kg})$ & 73.5 & 39.5 & 51.0 \\
BMI $\left(\mathrm{kg} / \mathrm{m}^{2}\right)$ & 29.3 & 18.0 & 23.4 \\
Body fat $(\%)$ & 38 & 19 & 34 \\
Bone age (y) & 14.1 & 12.2 & 14.1 \\
Peak Testosterone (ng/dl) (to hCG challenge) & 127.6 & 7.7 & 45.6 \\
Basal LH (mIU/ml) & 14.9 & 27.5 & 31.6 \\
Basal FSH (mIU/ml) & 33.7 & 76.2 & 82.2 \\
Peak GH (ng/ml) (to GHRH challenge) & 4.1 & 61.0 & 16.9 \\
IGF-1 (ng/ml) & 108.1 & 262.5 & 254.2 \\
BMD (DXA) (g/cm $\left.{ }^{2}\right)$ & 0.686 & 0.608 & 0.622 \\
\hline
\end{tabular}

BMI: body mass index, hCG: human chorionic gonadotropin, LH: luteinizing hormone, FSH, follicle stimulating hormone, GH: growth hormone, GHRH: GH releasing hormone, IGF-1: insulin-like growth factor-1, BMD: bone mineral density, DXA: dual energy x-ray absorptiometry.

causes were treated with $\mathrm{T}$ for pubertal induction. Case 1 had received irradiation to both testes and intensive chemotherapy for testicular relapse of acute lymphocytic leukemia at the age of $12 \mathrm{yr}$. Elevated basal gonadotropin levels and reduced $\mathrm{T}$ response to human chorionic gonadotropin (hCG) challenge led to the diagnosis of testicular dysfunction. GH insufficiency, evidenced by low $\mathrm{GH}$ response to GHRH challenge and a low serum IGF-1 level, were also recognized, although height was within the normal range. Case 2 had cryptorchidism and micropenis at birth with a 46XY karyotype. Repeated laparoscopic investigation did not detect the presence of gonads, but because Wolffian structures were present, he was diagnosed with vanishing testis syndrome. Case 3 had atrophic testes with cryptorchidism, hypospadias, and micropenis with a $46 \mathrm{XY}$ karyotype. His testes remained atrophic even when he entered puberty. His condition was categorized as embryonic testicular regression syndrome, although testes were recognized at birth. Cases 2 and 3 also had elevated basal gonadotropins and abolished responses of $\mathrm{T}$ to hCG stimulation, so that the diagnosis of hypogonadism was confirmed. Initial laboratory data are shown in Table 1. Bone age delay and elevated basal levels of gonadotropins with reduced T levels were constant findings. Obesity (body mass index $>25 \mathrm{~kg} / \mathrm{m}^{2}$ ) and/or increased body fat \% (>25), which are often observed in hypogonadal subjects, were observed in cases 1 and 3.

\section{Methods}

The subjects were treated with intramuscular $\mathrm{T}$ (testosterone enanthate $50 \mathrm{mg}$ ) every $4 \mathrm{wk}$. T treatment caused a rise in serum $\mathrm{T}$ levels in all subjects, although the values during treatment varied (24.7-189.6 ng/dl in case 1, 49.2-611.8 ng/ $\mathrm{dl}$ in case 2 , and 69.6-350.4 ng/dl in case 3). BMD and biochemical markers of bone turnover were measured at baseline, 6 mo and 1 yr of therapy. Lumbar BMD from L1 to L4 in the posterioranterior projection was evaluated by dual energy xray absorptiometry (DXA) (Hologic QDR-4500A, Hologic Inc., Waltham, MA), and mean values and SDS (standard deviation scores) were analyzed. 
Markers of bone turnover were measured with commercially available immunoassay kits. Serum osteocalcin (OC) was measured with immunoradiometric assay (BGP Mitsubishi, Tokyo, Japan); serum bone-specific alkaline phosphatase (bALP) (Metra BAP, Quidel, San Diego, CA), urinary deoxypyridinoline (Dpyr) (Metra DPD, Quidel), and urinary N-terminal telopeptide of type I collagen (NTx) (Osteomark, Mochida, Tokyo, Japan) were measured with enzyme-linked immunosorbent assay. Dpyr and NTx are expressed as per mg creatinine. All assays were performed at the Special Reference Laboratory (SRL) (Tokyo, Japan). All samples were obtained in the morning of regular visits. Informed consent was obtained from patients and their parents. The study was approved by the Ethical committee on human research of the Tohoku University School of Medicine.

\section{Statistical analysis}

Serial data for BMD and biochemical bone turnover markers were assessed by analysis of variance for two-factor repeated measures followed by Fisher's protected least significant difference method with correction for multiple comparisons as appropriate. Statistical significance was accepted at $p<0.05$. All values are expressed as the mean \pm S.D.

\section{Results}

\section{BMD}

Lumbar BMD before treatment $(0.65 \pm 0.06 \mathrm{~g} /$ $\mathrm{cm}^{2}$ ) was below $-2 \mathrm{SD}$ for age. BMD steadily increased in all cases during $\mathrm{T}$ therapy $(0.74 \pm 0.07$ $\mathrm{g} / \mathrm{cm}^{2}$ at 1 yr) (Fig. 1).

\section{Markers of bone turnover}

Markers of bone turnover before $\mathrm{T}$ replacement were higher than the adult range for the kits used in this study. Although normal values in children for these kits were not available, baseline levels for the subjects were roughly in the
BMD (SDS)

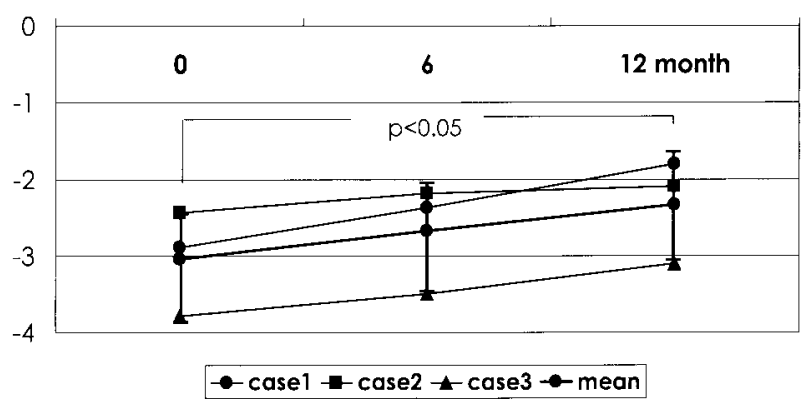

Fig. 1 Changes in lumbar bone mineral density (BMD) are shown. All cases and the mean \pm S.D. (bold) are shown.

prepubertal range according to data previously published (7-10). Dpyr and NTx were higher in case 2 than in the other subjects. All markers tended to increase by 6 mo, although this was statistically significant only in bALP and Dpyr (Fig. 2).

\section{Discussion}

Low BMD in hypogonadism is a constant finding in both adolescent boys and adults, and $\mathrm{T}$ replacement therapy causes an increase in BMD $(2-5)$. Our present study confirmed that the three adolescent hypogonadal boys had low BMD, and that $1 \mathrm{yr}$ of $\mathrm{T}$ therapy caused a steady increase in their lumbar BMD by 10 to $15 \%$.

Several studies $(3,4)$ in adults have shown that low BMD is associated with an increase in bone turnover markers in male hypogonadism. It has also been shown in adults that T therapy causes a reduction in resorption markers with an increase in BMD, whereas changes in bone formation markers are varied $(3,4,12)$. Therefore, in male hypogonadism, increased bone resorption appears to be a major contributor to low BMD, and mechanisms for an increase in BMD caused by $\mathrm{T}$ therapy are mainly due to suppressed bone resorption. This is in agreement with the finding that the effects of $\mathrm{T}$ therapy are largely due to those 

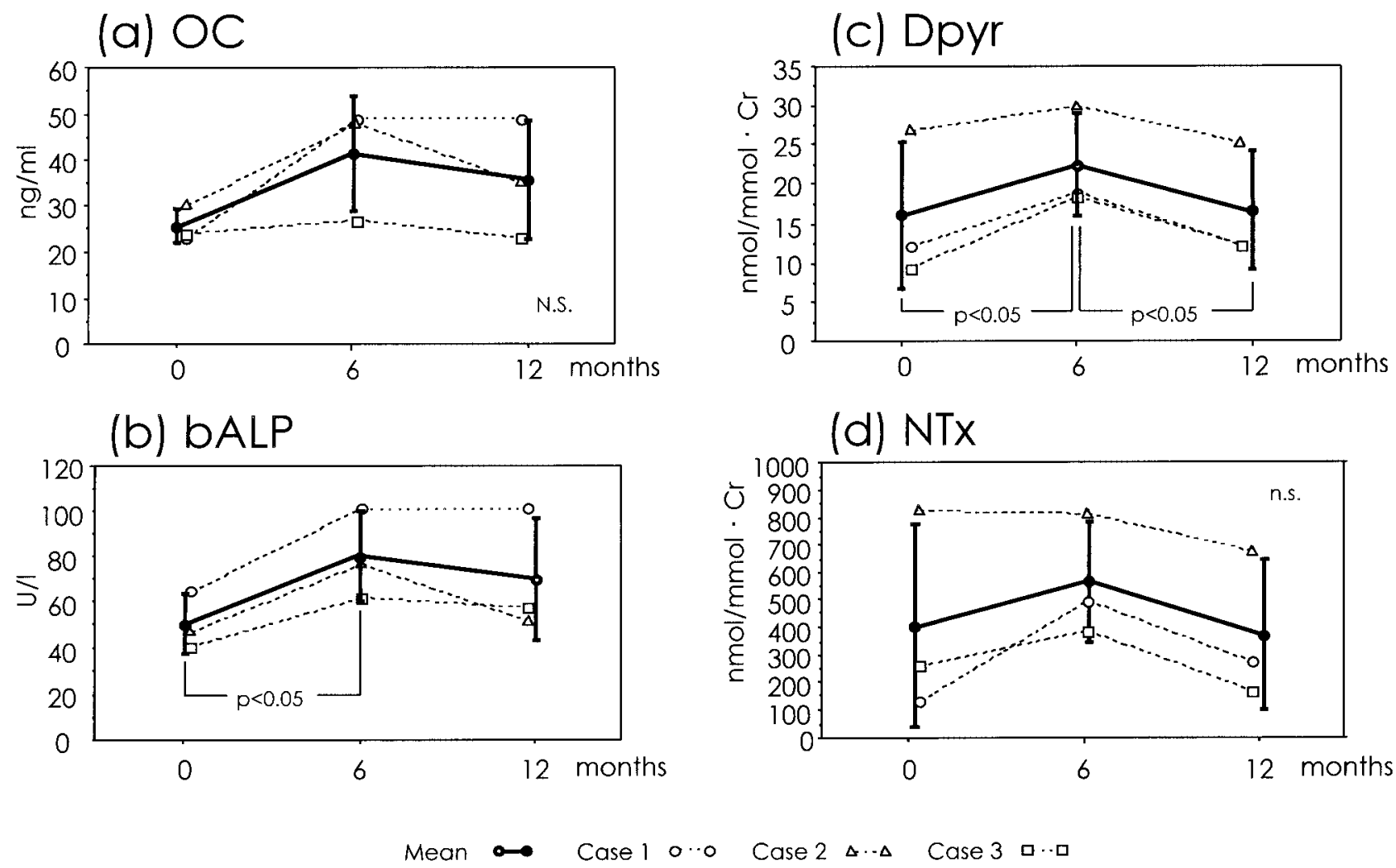

Fig. 2 Changes in biochemical markers of bone turnover before and during testosterone replacement. (a) OC: osteocalcin, (b) bALP: bone specific alkaline phosphatase, (c) Dpyr: deoxypyridinoline, (d) NTx: N-terminal telopeptide of type 1 collagen. N.S.: not significant. All cases and the mean \pm S.D. (bold) are shown.

of estrogens converted from $\mathrm{T}(6)$.

On the other hand, changes in bone turnover markers in boys with hypogonadism have not been described so far. Low BMD in adolescent hypogonadism is a consequence of the failure to gain bone mass during puberty, remaining in the prepubertal range, in contrast to adult-onset cases in which the BMD would have decreased after onset of the disease. Therefore, it is expected that bone turnover markers also remain in the prepubertal range in these young subjects. Markers for both bone formation and resorption, such as OC, bALP, Dpyr, and NTx, are much higher during childhood than in adults, with a further increase during puberty; levels then decrease to the adult range (7-11). The present study showed that these markers in the three hypogonadal boys are higher than the normal adult range, and they increased 6 mo after introduction of T therapy. These changes differ from those observed in adults, but are similar to those seen in spontaneous puberty, so that responses of skeletal markers to $\mathrm{T}$ administration appear to be different in younger subjects from those in older ones.

Reasons for the difference between younger and older subjects in response to $\mathrm{T}$ administration are not clear. One would suspect that the GH/IGF1 axis, which is activated during puberty and by exogenous $\mathrm{T}$ administration (13), might be involved, since GH administration results in an increase in markers of bone turnover $(14,15)$ Indeed, in the present study, the serum IGF-1 level increased in all subjects during the therapy including case 1 with GH insufficiency (108.1 to 
$221.9 \mathrm{ng} / \mathrm{ml}$ in case $1,262.5$ to $379.6 \mathrm{ng} / \mathrm{ml}$ in case $2,254.2$ to $350.8 \mathrm{ng} / \mathrm{ml}$ in case 3 ). It is also possible that the immature skeleton acts differently from the mature one in response to the same anabolic stimuli.

In conclusion, $\mathrm{T}$ administration in the hypogonadal adolescents caused a steady increase in BMD. This was accompanied by an initial increase in markers for both bone formation and resorption in contrast to older subjects in whom these are generally suppressed or unaltered. These changes are similar to those observed in spontaneous puberty, and may be due to an exaggerated $\mathrm{GH} / \mathrm{IGF}-1$ axis, or to differential responsiveness of the growing skeleton to anabolic stimuli.

\section{References}

1. Soyka LA, Fairfield WP, Klibanski A. Hormonal determinants and disorders of peak bone mass in children. J Clin Endocrinol Metab 2000;85:395163.

2. Arisaka O, Arisaka M, Nakayama Y, Fujiwara S, Yabuta K. Effect of testosterone on bone density and bone metabolism in adolescent male hypogonadism. Metabolism 1995;44:419-23.

3. Katznelson L, Finkelstein JS, Schoenfeld DA, Rosenthal DI, Anderson EJ, Klibanski A. Increase in bone density and lean body mass during testosterone administration in men with acquired hypogonadism. J Clin Endocrinol Metab 1996;81:4358-65.

4. Guo CY, Jones TH, Eastell R. Treatment of isolated hypogonadotropic hypogonadism: Effect on bone mineral density and bone turnover. J Clin Endocrinol Metab 1997;82:658-65.

5. Finkelstein JS, Klibanski A, Neer RM, Doppelt SH, Rosenthal DI, Segre GV, et al. Increases in bone density during treatment of men with idiopathic hypogonadotropic hypogonadism. J Clin Endocrinol Metab 1989;69:776-83.

6. Farahati-Nini A, Riggs BL, Atkinson EJ, O'Fallon WM, Eastell R, Khosla S. Relative contributions of testosterone and estrogen in regulating bone resorption and formation in normal elderly men. $\mathrm{J}$
Clin Invest 2000;106:1553-60.

7. Kanzaki S, Hosoda K, Moriwake T, Tanaka H, Kubo $\mathrm{T}$, Inoue M, et al. Serum propeptide and intact molecular osteocalcin in normal children and children with growth hormone (GH) deficiency: a potential marker of bone growth and response to GH therapy. J Clin Endocrinol Metab 1992;75:1104-9.

8. Bollen AM, Eyre DR. Bone resorption rates in children monitored by the urinary assay of collagen type I cross-linked peptides. Bone 1994;15:31-4.

9. Fujimoto S, Kubo T, Tanaka H, Miura M, Seino Y. Urinary pyridinoline and deoxypyridinoline in healthy children and in children with growth hormone deficiency. J Clin Endocrinol Metab 1995;80:1922-8.

10. Tobiume H, Kanzaki S, Hida S, Ono T, Moriwake T, Yamauchi S, et al. Serum bone alkaline phosphatase isozyme levels in normal children and children with growth hormone (GH) deficiency: a potential marker for bone formation and response to GH therapy. J Clin Endocrinol Metab 1997;82:2056-61.

11. Szulc P, Seeman E, Delmas PD. Biochemical measurements of bone turnover in children and adolescents. Osteoporos Int 2000;11:281-94.

12. Wang C, Eyre DR, Clark R, Kleinberg D, Newman $\mathrm{C}$, Iranmanesh A, et al. Sublingual testosterone replacement improves muscle mass and strength, decreases bone resorption, and increases bone formation markers in hypogonadal men - a clinical research center study. J Clin Endocrinol Metab 1996;81:3654-62.

13. Kerrigan JR, Rogol AD. The impact of gonadal steroid action on growth hormone secretion during childhood and adolescence. Endocr Rev 1992;13:281-98.

14. Crofton PM, Sterling HF, Schonau E, Kelnar CJH. Bone alkaline phophatase and collagen markers as early predictors of height velocity response to growth-promoting treatment in short normal children. Clin Endocrinol 1995;44:385-94.

15. Nilsson AG. Effects of growth hormone replacement therapy on bone markers and bone mineral density in growth hormone-deficient adults. Horm Res 2000;54 (Suppl 1):52-7. 